\title{
Social Belief Revisioning in Multi-Agent System on the basis of Social and Moral Factors
}

\author{
Jasdeep Kaur \\ Student of M.Tech. CSE (4th sem ), \\ Guru Nanak Dev University, Regional campus, \\ Gurdaspur
}

\author{
Harjot Kaur \\ Assistant Professor \\ Department of computer science and engineering, \\ Guru Nanak Dev University, Regional campus, \\ Gurdaspur
}

\begin{abstract}
The organization will be affected by the presence of employees. The dedication of employees will decide whether organization will earn profit or not. The simulative environment is constructed in the proposed paper using Netlogo in order to analyse impact of agents within the organization. The belief revisioning is considered as a factor of altering the faith of the agents due to which agents may alter their behaviour hence impacting the growth of organization. The simulative environment also suggests the ways by which beliefs of agents are revised. The case study on market research is considered in the proposed paper.
\end{abstract}

\section{Keywords}

Belief Revisioning, Agents, Organization, Multi-agent systems

\section{INTRODUCTION}

Belief revisioning is a fundamental process that underlies numerous forms of intelligent behaviour. The intelligent information systems which are in fashion nowadays must make sure that they can modify their beliefs in a reasonable, coherent and compatible fashion. [1] It is shown that standard relational database technology can be used to implement iterated belief revision strategies which is a major progress in technology. For the purpose of motivation, a problem in market research, namely modelling changes to consumer priorities will be taken into notice. The major idea is that possible product profiles can be represented as tuples in a relational database, and the consumer's priorities for products is captured using an ordinal ranking over the tuples. Using this representation, iterated belief revision policies can be implemented simply and efficiently. In particular, belief revisioning is performed using a technique named database transactions that will modify this ranking.

The online shopping sites generally utilize belief revision mechanisms in order to divert the users toward their websites. This will help in increasing the user database and hence earn profit. The online websites generally consider market research to ensure that maximum number of users are interacted towards them. Belief revisioning is what is used in that context. The model will have to be constructed ensuring the satisfaction of suggested technique. [2] The two major factors that also play critical role in revisioning of the belief of the agents are priority and relevance. The Advanced growth model suggested in the references paper ensures that belief revisioning results in benefit of the organization. The market research conducted in order to calculate the percentage of users impacted through AGM model eventually leads to belief revisioning of the agents. The Advanced Growth Model calculates the size of community which is impacted by certain parameters such as caste, creed etc and form clusters to determine strong parameters out of many parameters which influence the agents.

\subsection{The problem of belief revision}

Marketing research is the mechanism by which belief of the agents involved can be easily analysed. The analysis of belief revisioning is determined using large number of parameters. The belief refining process will be described by considering the following example [3] Suppose there exist a database that contains among other things, information about the swans. The constraints are listed as

$\alpha:$ All European swans are Black

$\beta$ : All Black swans comes from Europe.

$¥:$ The birds trapped appears from Europe

$\oint:$ Sweden is a part of Europe.

If the database is attached and logical inference is made then it is concluded that

$£$ : The caught birds are black.

Suppose if a bird comes out to be white, then negation of the beliefs present within the database is done, but this will make the database inconsistent. So in order to solve the problem in a distinguished way, we may need to revise the beliefs that are present within the database. The problem of belief space updating is a logical mechanism. Every updation to the belief space has some consequence that is associated with it. Also logical consequence that we have attained will not indicate which belief to be updated. So it is a matter of making cautious decision in deciding which belief to be updated and which belief should remain immobile.

\subsection{Representation of beliefs in database}

The beliefs in database are represented in the form of rules and facts. The rules of standard logical consequence may be followed while using beliefs. The conclusion derived from the statements is closely related to the utilized facts. In some cases the elements described explicitly in the database have a special status in comparison to the logical consequences of the beliefs that were derived by some inference mechanism. In other cases, the derivation of the beliefs in the database is immaterial so that any representation of the beliefs that has the same logical consequences, i.e., the same set of implicit beliefs, is equivalent. As we will see in lot of others papers in this volume, the nature of the relation between explicit and implicit beliefs is of specific importance for how the belief revision process is attacked.

Logic alone is not a single thing that will decide which beliefs to give up and which to retain when performing a belief revisioning mechanism. We must also discover the extra logical factors that determine the choices. One mechanism is 
that the information lost when removing certain beliefs should be kept minimum to a certain extent. One more idea is that some beliefs are considered more important than others and the beliefs that should be given up are the least important ones. In this era of computer science, there is the most common solution of handling the problem which is discovered and that is the use of integrity constraints. Again, the methodological rules chosen here are dependent on the application area.

\subsection{Types of belief revision}

We define that a belief revision process occurs only when a new piece of information or logics that is inconsistent with the present belief system (or database) is added to the present system in such a way that the result is a new consistent belief system. [1], [2], [4]-[18]But this is not the only kind of change that can occur in a belief system. Different typologies of belief changes are possible in the present belief system depending on how beliefs are represented and what kinds of inputs are accepted in that system. In the most general case, when beliefs are represented by sentences in some code, and when a belief is either accepted or rejected in a belief system, say K (so that no degrees of belief are considered), one can define three main kinds of belief changes :

i. Expansion : Whenever a new sentence $\varphi$ is added to a belief system $\mathrm{K}$ along with all the logical consequences of the addition, it does not make sure that the larger set found is inconsistent. The belief system that results from expanding $\mathrm{K}$ by a sentence $\varphi$ added will be denoted by :

$$
\mathrm{K}+\varphi
$$

ii. Revision: Now we add a new sentence that is inconsistent with a belief system $\mathrm{K}$, but, in order to maintain consistency in the resulting belief system, some of the old sentences in $\mathrm{K}$ must be deleted. The result of revising $\mathrm{K}$ by a sentence $\varphi$ will be denoted by:

\section{$\mathrm{K} \varphi$}

iii. Contraction: Some sentence in $\mathrm{K}$ are retracted without adding any new facts. In order for the resulting system to be closed under the operation of logical consequences, some other sentences from belief system $\mathrm{K}$ must be given up. The result of contracting $\mathrm{K}$ with respect to $\varphi$ will be denoted by:

$$
\mathrm{k}-\varphi
$$

\section{PROPOSED ALGORITHM}

The proposed algorithm utilizes multiple factors belief revision model.

1) Agent $\left(\mathrm{A}_{\mathrm{i}}\right)=$ Organization $_{\mathrm{i}}$ where $\mathrm{i}=0$ to $\mathrm{n}$

2) Set the $\operatorname{Belief}\left(B_{i}\right)=\left\{B_{1}, B_{2},-----, B_{n}\right\}$

3) Compare $B_{i}$ with +ve influence if True Remains within the

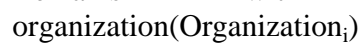

Modify $\mathrm{B}_{\mathrm{i}}$

Else

Shift the Organization $\left(\right.$ Organization $\left._{\mathrm{i}}\right)$

4) stop

The above algorithm utilizes two phase belief revisioning which is described in the next section.

\subsection{Two approaches for belief revisioning}

The belief revisioning process in the proposed system is divided into two parts. The first part considers the social factors in the revisioning of the belief. The social factors will include religion, caste or wealth. One agent will act as an influencer and that influencer agent will influence the agents through the social factors and consequently groups of similar batches will be formed. The experimented results are shown as follows :

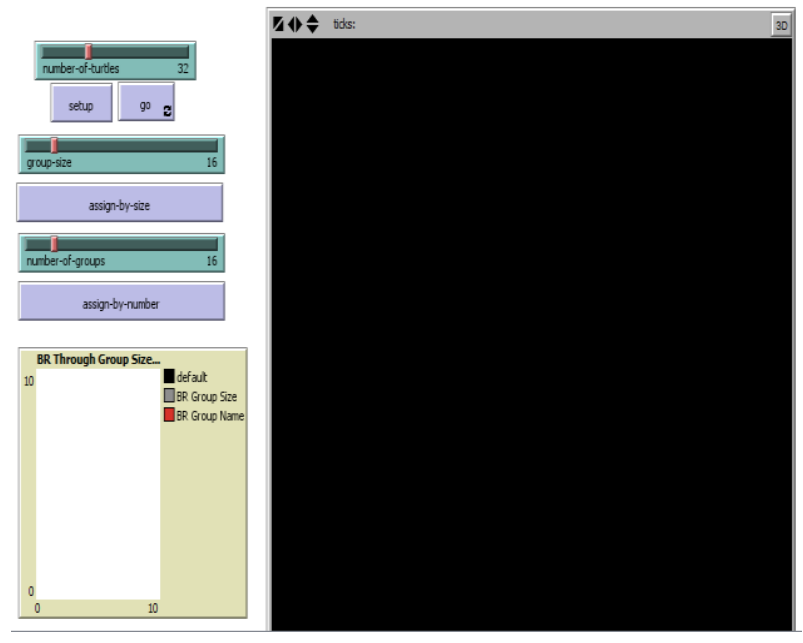

Fig 1 : Setup screen for belief revisioning mechanism using Netlogo

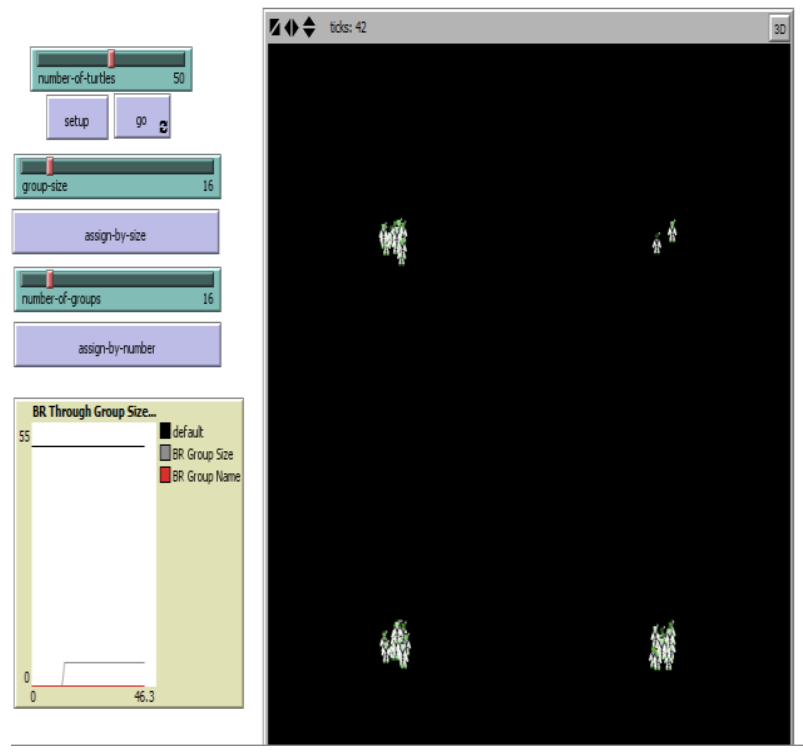

Fig 2 : Belief revisioning shown on the basis of social factors

In experimentation, it is represented in the form of Group Size. The mathematical formulation which is used is as follows :

\section{$S G_{i}=\alpha * \sum($ Caste $V$ Wealth $V$ Religion $)$}

$\mathrm{SG}_{\mathrm{i}}$ indicate social group, $\alpha$ is constant determining strength to which person belongs to this group or not. The values of $\alpha$ lie in between 0 and 1 .

The second way to distinguish between the beliefs is on the basis of right and wrong identification capacity which are also known as moral factors. The moral factors can also partition the agents. The experimented results are represented as : 

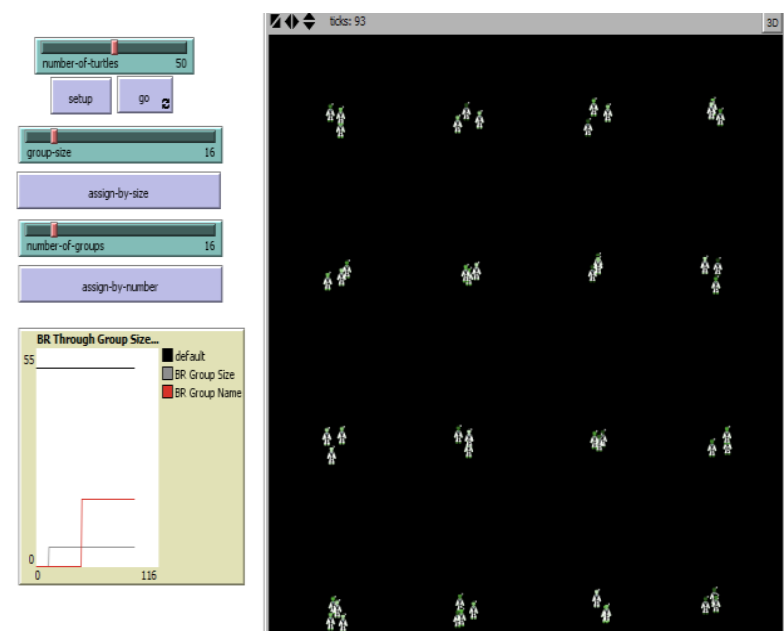

Fig 3 : Belief revisioning shown on the basis of Moral Values

The belief revisioning on the basis of moral values will be represented in the experimentation through assignment by numbers. The mathematical representation for moral factors is as follows :

$$
M F_{i}=\beta * \sum(\text { Right } V \text { Wrong })
$$

The equation contains $\beta$ which indicates strength lying between 0 and 1 . The moral factors are based on the fact that whether the things are right and wrong and groups are formed according to those beliefs.

\section{CONCLUSION AND FUTURE SCOPE}

The belief revisioning can be accomplished through number of factors. In the proposed paper only two of such parameters are considered. The belief revisioning process on the basis of moral and social aspects results in two distinct groups which will identify the attributes possessed by the agents within the group. The future scope of this revisioning is that the cluster of agents hence identified can then be utilized for enhanced task and can help organization in moving to high profit state. So, we can help the organization to attain profits using the two factors above. In future, this revisioning can be practically implemented using Artificial Intelligence and with the help of supercomputers. Till then this will remain as a theoretical concepts of belief revisioning. The mathematical formulation of result is also suggested describing the multi facet aspects of agents within the organization.

\section{REFERENCES}

[1] M.-A. Williams, "Belief revision as database update," in Proceedings Intelligent Information Systems. IIS'97, pp. 410-414.

[2] M. Wu, "Priority and relevance in belief revision," in 2011 6th IEEE Joint International Information Technology and Artificial Intelligence Conference, 2011, vol. 1, pp. 459-463.

[3] S. Tojo, "Collective belief revision in linear algebra," pp. $175-178$.
[4] G. D. I. Luna, Y. M. Martinez, and L. C. A. Robles, "Applying Max-2SAT to Efficient Belief Revision," in 2011 10th Mexican International Conference on Artificial Intelligence, 2011, pp. 9-15.

[5] A. F. Dragoni and P. Puliti, "Distributed belief revision versus distributed truth maintenance," in Proceedings Sixth International Conference on Tools with Artificial Intelligence. TAI 94, pp. 499-505.

[6] R. Y. K. Lau, B. Essam, and S. Y. Chan, "Belief revision for adaptive negotiation agents," in IEEE/WIC International Conference on Intelligent Agent Technology, 2003. IAT 2003., pp. 196-202.

[7] S. M. Sripada, "A temporal approach to belief revision in knowledge bases," in Proceedings of 9th IEEE Conference on Artificial Intelligence for Applications, pp. 56-62.

[8] H. Decker and R. De Juan-Marin, "InconsistencyTolerant Belief Revision for Distributed Decision Support," in 2013 Eighth International Conference on P2P, Parallel, Grid, Cloud and Internet Computing, 2013, pp. 387-393.

[9] O. Doukari, E. Wurbel, and R. Jeansoulin, "A New Model for Belief Representation and Belief Revision Based on Inconsistencies Locality," in 19th IEEE International Conference on Tools with Artificial Intelligence(ICTAI 2007), 2007, vol. 2, pp. 262-269.

[10] A. Kini and J. Choobineh, "Trust in electronic commerce: definition and theoretical considerations," in Proceedings of the Thirty-First Hawaii International Conference on System Sciences, vol. 4, pp. 51-61.

[11] B. M. DePaulo, J. J. Lindsay, B. E. Malone, L. Muhlenbruck, K. Charlton, and H. Cooper, "Cues to deception," Psychol. Bull., vol. 129, no. 1, pp. 74-118, 2003.

[12] P. Date, "Encryption in the Cloud," no. April, pp. 1547$1551,2014$.

[13] B. A. R, V. A. A, L. M. P, P. A. B, and M. Westley, "Forecasting Model for Criminality in Barangay Commonwealth, Quezon City, Philippines using Data Mining Techniques," vol. 3, pp. 28-33, 2015.

[14] N. Dubey and S. K. Chaturvedi, "A Survey Paper on Crime Prediction Technique Using Data," vol. 4, no. 3, pp. 396-400, 2014.

[15] A. Morris, W. Ross, and M. Ulieru, "Modelling culture in multi-agent organizations," Adv. Agent Technol., vol. 7068, pp. 65-79, 2012.

[16] M. Systems, A. Morris, W. Ross, H. Hosseini, and M. Ulieru, "Modeling Culture with Complex ," Science (80).

[17] J. T. Hancock, "Digital Deception: Why, When and How People Lie Online," Oxford Handb. Internet Psychol., pp. 289-301, 2007. 